\title{
Affected Body Area
}

National Cancer Institute

\section{Source}

National Cancer Institute. Affected Body Area. NCI Thesaurus. Code C66994.

A part of the body showing particular characteristics. 